\title{
Transparencia en tres dimensiones
}

\author{
Frederick Schauer*
}

\begin{abstract}
RESUMEN
El artículo aborda el problema de la transparencia en la toma de decisiones públicas, of reciendo un marco que permite evaluar los objetivos y principios asociados con la transparencia, con sus costos y beneficios y cómo la transparencia está conectada con otros principios, como la libertad de expresión. El texto discute la definición de transparencia: el grado de transparencia depende de tres variables, cuales son el poseedor de la información, la información que debe ser transparentada y quién debe tener acceso a la información. Luego, el texto aborda los objetivos de la transparencia, en particular, los fines regulatorios, la promoción de la democracia, la promoción de la eficiencia $y$ los fines epistemológicos. El artículo denota que la transparencia es conservadora, al intentar prevenir los peores escenarios incluso al costo de prevenir la adopción de mejores decisiones.
\end{abstract}

Transparencia - libertad de expresión - rendición de cuentas

\section{Transparency in three dimensions}

\begin{abstract}
The paper addresses the issue of transparency in public decision making, offering a proposed framework for assessing the goals and benefits, and how transparency is related to other principles, including those of the First Amendment. The text discusses the definition of transparency: the degree of transparency is a function of three variables: the possessor of information, the information that is to be made transparent, and to whom access to information will be given. Then, it addresses the aims of transparency, in particular, its regulatory, democracy enhancing, efficiency promoting, and epistemological goals. The text notes how transparency is conservative, seeking to prevent the worst outcomes even at the occasional cost of foreclosing the best ones.
\end{abstract}

Transparency - free speech - accountability

* David and Mary Harrison Distinguished Professor of Law, University of Virginia.

Este texto original corresponde a la versión escrita y anotada de la David C. Baum Memorial Lecture on Civil Rights and Civil Liberties dictada el 11 de noviembre de 2010 en la Facultad de Derecho de la Universidad de Illinois.

La traducción, desde el inglés, corresponde al artículo "Transparency in Three Dimensions", publicado en University of Illinois Law Review (Vol. 2011, No 4). Ha sido traducido por Raúl Letelier W. y Pablo Contreras V., ambos profesores de la Facultad de Derecho de la Universidad Alberto Hurtado.

Artículo recibido el 30 de marzo de 2014 y aceptado para su publicación por el Comité Editorial el 28 de mayo de 2014. 
$\mathrm{L}$ a invitación que me ha hecho la Facultad de Derecho de la Universidad de Illinois para dictar la David C. Baum Memorial Lecture on Civil Rights and Civil Liberties (Clase Magistral David C. Baum sobre Derechos y Libertades Civiles) constituye para mí un inmenso honor. Esta serie de conferencias conmemora la vida y logros de un gran académico y una excelente persona quien representa una parte importante de la historia de esta Facultad. Me siento honrado al incorporarme a un listado tan distinguido de oradores en las Baum Lectures, algunos de ellos mis amigos y otros que gozan de una reputación que ni siquiera en sueños puedo aspirar.

Varias de las anteriores Baum Lectures, especialmente en los primeros años de la serie, estuvieron dedicadas a los logros de personajes determinados, especialmente aquellos eminentes jueces de la Corte Suprema -Hugo Black ${ }^{1}$, Oliver Wendell Holmes ${ }^{2}$, Louis Brandeis $^{3}$, Robert Jackson ${ }^{4}$, Felix Frankfurter ${ }^{5}$, William Douglas ${ }^{6}$, Thurgood Marshall ${ }^{7}$ y William Brennan ${ }^{8}$-, cuyo trabajo desarrolló la tradición estadounidense de derechos y libertades civiles. Pese a ello, la focalización en el trabajo de personas determinadas no ha sido una tradición sin quiebres ${ }^{9}$. Lo digo afortunadamente para mí, porque quiero hablarles no sobre una persona en específico, sino sobre una idea. Y la idea de la que quiero hablarles es de transparencia.

La transparencia, en estos días, está en todas partes, o, al menos, se habla de ella en cualquier lugar ${ }^{10}$. En efecto, no hace un mes el profesor de esta Facultad Lawrence

${ }^{1}$ John P. Frank, Hugo L. Black: Free Speech and the Declaration of Independence, 1977 U. ILL. L.F. 577.

${ }^{2}$ Grant Gilmore, Some Reflections on Oliver Wendell Holmes, Jr., 2 GREEN BAG 2D 379 (1999).

${ }^{3}$ Nathaniel L. Nathanson, The Philosophy of Mr. Justice Brandeis and Civil Liberties Today, 1979 U. ILL. L.F. 261.

${ }^{4}$ Philip B. Kurland, Justice Robert H. Jackson-Impact on Civil Rights and Civil Liberties, 1977 U. ILL. L.F. 551.

${ }^{5}$ William T. Coleman, Jr., Mr. Justice Felix Frankfurter: Civil Libertarian As Lawyer and As Justice: Extent to Which Judicial Responsibilities Affected His Pre-Court Convictions, in Six Justices on Civil Rights 85 (Ronald D. Rotunda ed., 1983).

${ }^{6}$ Vern Countryman, Justice Douglas and Freedom of Expression, 1978 U. ILL. L.F. 301.

${ }^{7}$ Mark V. Tushnet, The Jurisprudence of Thurgood Marshall, 1996 U. ILL. L. REV. 1129.

${ }^{8}$ Abner J. Mikva, Justice Brennan and the Political Process: Assessing the Legacy of Baker v. Carr, 1995 U. ILL. L. Rev. 683.

9 “Afortunadamente”, en especial, porque la tradición -tal como fue ejemplificada en las 8 previas Clases de Baum recién citadas- ha sido marcada por la práctica de exasistentes de jueves escribiendo con admiración sobre jueces a los cuales han servido. No habiendo sido un asistente de juez, soy incapaz de ejecutar tal rol.

10 Among many prominent recent writings, see generally Archon fung et al., Full dis-closure: the perils and promise of transparency (2007); open government: collaboration, transparency, and participation in practice (Daniel Lathrop \& Laurel Ruma eds., 2010); Cary Coglianese et al., Transparency and Public Participation in the Federal Rule- making Process: Recommendations for the New Administration, 77 Geo. Wash. L. Rev. 924 (2009); Cynthia Estlund, Just the Facts: The Case for Workplace Transparency, 63 Stan. L. Rev. 351 (2011); Kenneth Feinberg, Transparency and Civil Justice: The Internal and External Value of Sunlight, 58 Depaul L. Rev. 473 (2009); Mark Fenster, Designing Transparency: The 9/11 Commission and Insti- tutional Form, 65 Wash. \& Lee L. Rev. 1239 (2008); Mark Fenster, Seeing the State: Transparency As Metaphor, 62 Admin. L. Rev. 671 (2010); Eugene R. Fidell, Transparency, 2009, 61 Hastings L.J. 457 (2009); William Funk, Public Participation and Transparency in Administrative Law-Three Examples As 
Solum discutía el tópico de la transparencia en su blog de Teoría Jurídica, precisamente, porque él consideraba que era un concepto del cual los estudiantes de primer año debían tener conciencia ${ }^{11}$. El profesor Solum está en lo correcto, de hecho, más en lo correcto de lo que podría haber estado hace una década. El presidente Obama ha prometido explícitamente un gobierno más transparente y, desde el inicio, la Administración Obama ha dictado instrucciones con el fin de implementar dicha promesa ${ }^{12}$. Reformas regulatorias recientes han accedido a las demandas de defensores de los consumidores que instaban por más transparencia en hipotecas, créditos, actividades bancarias y otras operaciones financieras ${ }^{13}$. Por su parte, defensores de accionistas insisten en que las sociedades deben ser más transparentes tanto en el gobierno como en sus decisiones corporativas ${ }^{14} \mathrm{y}$, simultáneamente, las mismas empresas junto a otros actores urgen por mayor transparencia como alternativa frente a una supuesta regulación de "mano dura" 15 . Los tribunales, que con sus audiencias abiertas, acceso público a sus expedientes y escrituración de sus razones se encuentran entre las instituciones más transparentes para la toma de decisiones, son presionados a aumentar aquella transparencia ${ }^{16}$. Los promotores de tecnología computacional de "código abierto" condenan las leyes y contratos que

an Object Lesson, 61 admin. L. Rev. 171 (2009); Jacob E. Gersen \& Anne Joseph O’Connell, Hid- ing in Plain Sight? Timing and Transparency in the Administrative State, 76 U. CHI. L. REV. 1157 (2009); Seth F. Kreimer, The Freedom of Information Act and the Ecology of Transparency, 10 U. PA. J. CONST. L. 1011 (2008); Lynn M. LoPucki, Court-System Transparency, 94 Iowa L. Rev. 481 (2009); Michael R. Siebecker, Trust \& Transparency: Promoting Efficient Corporate Disclosure Through Fiduciary-Based Discourse, 87 Wash. U.L. REV. 115 (2009).

${ }^{11}$ Lawrence B. Solum, Legal Theory Lexicon: Transparency, Legal Theory Blog (Oct. 3, 2010, 11:00 AM), http://solum.typepad.com/legaltheory/2010/10/legal-theory-lexicon-transparency. html.

12 Presidential Memorandum on Freedom of Information Act, 74 Fed. Reg. 4683 (Jan. 26, 2009); Presidential Memorandum on Transparency and Government, 74 Fed. Reg. 4685 (Jan. 26, 2009); Memorandum from Peter R. Orszag, Dir., Office of Mgmt. and Budget, to the Heads of Exec. Dep'ts and Agencies (Dec. 8, 2009), http://www.whitehouse.gov/sites/default/files/omb/assets/mem oranda_2010/ m10-06.pdf. Se mantiene controvertido el grado de cumplimiento de esta promesa por la Administración de Obama. Véase Nat'l security archive, sunshine and shadows: the clear Obama message for freedom of information meets mixed results (2010), available at http://www.gwu.edu/ nsarchiv/NSAEBB/NSAEBB308/index.htm. Sobre transparencia en el gobierno en general, véase Elizabeth Garrett et al., Constitutional Issues Raised by the Lobbying Disclosure Act, in The lobbying manual: a complete guide to federal lobbying law and practice 197 (William V. Luneburg et al., eds., 4th ed. 2009); Transparency: The Key To Better Governance? (Christopher Hood \& David Heald eds., 2006); Danielle Keats Citron, Open Code Governance, 2008 U. Chi. Legal F. 355.

13 Véase Diana Golobay, Fed Publishes Wave of Rules for Mortgage Origination Transparency, Housingwire (Aug. 16, 2010, 12:01 PM), http://www.housingwire.com/2010/08/16/fed_publishes_ wave_of_rules_for_mortgage_origination_transparency.

${ }^{14}$ Un análisis ponderado de estas cuestiones, mostrando tanto los beneficios como los costos de la transparencia en gobiernos corporativos, se encuentra en Benjamin E. Hermalin \& Michael S. Weisbach, Transparency and Corporate Governance (Univ. of Cal. \& Nat'l Bureau of Econ. Research, Working Paper No. W12875, 2007), available at http://papers.ssrn.com/sol3/papers.cfm?abstract_id=958628.

${ }^{15}$ Véase, v.gr., L. Gordon Crovitz, Op.-Ed., Transparency is More Powerful Than Regulation, WALL ST. J., Mar. 30, 2009, at A21; Richard H. Thaler \& Cass R. Sunstein, Op.-Ed., Disclosure is the Best Kind of Credit Regulation, Wall ST. J., Aug. 13, 2008, at A17.

${ }^{16}$ Véase LoPucki, supra note 10; véase también Symposium, Tradeoffs of Candor: Does Judicial Transparency Erode Legitimacy?, 64 N.Y.U. ANN. Surv. AM.L. 443 (2009). 
exaltan la propiedad por sobre la transparencia ${ }^{17}$. Por otro lado, la divulgación se alza como la piedra angular en numerosos otros aspectos del derecho, ya sea en el contexto del consentimiento informado ${ }^{18}$, la regulación de títulos de valor ${ }^{19}$, o en las advertencias de Miranda ${ }^{20}$ que la policía hace a aquellos que interroga. Finalmente, incluso-o, quizás debiésemos decir, especialmente- en la arena internacional, agrupaciones de derechos humanos como Transparencia Internacional ${ }^{21}$, las fundaciones Open Society ${ }^{22}$ y otras con nombres menos literalmente transparentes tratan a la transparencia gubernamental como uno de sus objetivos centrales ${ }^{23}$.

La transparencia no es, por cierto, un bien puro, pese a la vasta retórica popular contemporánea ${ }^{24}$. Cuando le pedimos a un amigo guardar un secreto ${ }^{25}$, cuando una decisión es tomada para favorecer la protección de la privacidad, en cada caso en que

${ }^{17}$ Véase en general John R. Ackermann, Toward Open Source Hardware, 34 U. Dayton L. Rev. 183 (2009); Katie Fortney, Ending Copyright Claims in State Primary Legal Materials: Toward an Open Source Legal System, 102 L. Libr. J. 59 (2010); Michael J. Madison et al., Constructing Commons in the Cultural Environment, 95 Cornell L. Rev. 657 (2010); Greg R. Vetter, Open Source Licensing and Scattering Opportunism in Software Standards, 48 B.C. L. Rev. 225 (2007).

${ }^{18}$ Véase Joseph Leghorn et al., The First Amendment and FDA Restrictions on Off-Label Uses: The Call for a New Approach, 63 Food \& Drug L.J. 391, 405-06 (2008); Richard S. Saver, At the End of the Clinical Trial: Does Access to Investigational Technology End As Well?, 31 W. New Eng. L. Rev. 411,447 (2009).

${ }^{19} \mathrm{La}$ aproximación convencional a la publicidad en material de títulos de valor es descrita y desafiada en Susanna Kim Ripken, The Dangers and Drawbacks of the Disclosure Antidote: Toward a More Substantive Approach to Securities Regulation, 58 Baylor L. Rev. 139, 151 (2006).

${ }^{20}$ Miranda v. Arizona, 384 U.S. 436 (1966).

Nota de los traductores: Una de las más importantes consecuencias de este caso fue la modificación en las reglas procedimentales para casos de detenciones. La llamada "Advertencia de Miranda" es un set de derechos (derecho a guardar silencio, derecho a tener un abogado y advertido de que todo lo que se diga puede ser usado en el procedimiento penal) que todos los oficiales de policía deben leer antes de cualquier detención.

${ }^{21}$ Véase Transparency Int'l, http://www.transparency.org (last visited May 16, 2011).

${ }^{22}$ Véase Open Society Found., http://www.soros.org (last visited May 16, 2011).

${ }^{23}$ Véase, v. gr., Article 19, Changing The climate for freedom of expression and Miranda V. Arizona, 384 U.s. 436 (1966). Véase Transparency Int'l, http://www.transparency.org (last visited May 16, 2011). Véase Open Society Found., http://www.soros.org (last visited May 16, 2011). Freedom of Information (2009), http://www. article19.org/pdfs/publications/changing-the-climate-for-freedom-of-expression-and-freedom-of-information. pdf; Article 19, the London declaration for transparency, the free flow of information and development (2010), http:// www.right2info-mdgs.org/wp-content/uploads/London-Declaration.pdf.

${ }^{24}$ En efecto, análisis escépticos sobre las supuestas virtudes de la transparancia han ido apareciendo con más frequencia. Véase, v. gr., Omri Ben-Shahar \& Carl E. Schneider, The Failure of Mandated Disclosure, 159 U. PA. L. Rev. 647 (2011); Amitai Etzioni, Is Transparency the Best Disinfectant?, 18 J. POL. PHIL. 389 (2010); Florencia Marotta-Wurgler, Does Disclosure Matter? (NYU Law and Economics, Research Paper $\mathrm{N}^{\circ}$ 10-54, 2010), available at http://papers.ssrn.com/sol3/papers.cfm?abstract_id=1713860.

${ }^{25}$ El clásico y todavía altamente valioso tratamiento del secreto se encuentra en Sissela bok, secrets: on the ethics of concealment and revelation (1982). Un análisis más reciente, distinguiendo importantemente entre secreto conocido y desconocido, en David E. Pozen, Deep Secrecy, 62 Stan. L. Rev. 257 (2010). 
se valora el anonimato ${ }^{26}$ o cuando la prensa insiste en el secreto de sus fuentes, ${ }^{27}$ por ejemplo, se hacen peticiones válidas que representan, en realidad, reclamos por menos transparencia. Secreto, privacidad, anonimato y confidencialidad tienen también sus virtudes, las que nos permiten entender por qué la transparencia es un atributo más deseable en una terraza con ventanales que en las puertas de los baños. En ocasiones, parece que la transparencia es el principal ejemplo de aquel viejo adagio que dice que la perspectiva con que se mire depende del lugar donde estemos sentados ${ }^{28^{*}}$.

A pesar que las virtudes y vicios de la transparencia bien valen el esfuerzo de ser debatidas y analizadas $-\mathrm{y}$ de hecho lo han sido de manera creciente en años recientes ${ }^{29}$-; mi objetivo aquí es dar un paso atrás de estas discusiones y tratar de comprender qué se supone que conlleva la transparencia y cómo esta se encaja en temáticas más amplias sobre el rol del Derecho en la producción del conocimiento.

Mi objetivo en esta oportunidad es explorar y explicar tres diferentes aspectos del problema de la transparencia. El primero dice relación con la multiplicidad de objetivos que la transparencia busca cumplir. El segundo se refiere a la naturaleza peculiarmente conservadora - en el sentido apolítico del término- de la transparencia. El tercero, para finalizar, intenta situar parte de la discusión sobre la transparencia al lado de las más venerables tradiciones de libertad de expresión y de prensa, incluyendo, pero no limitándome a exponer mis opiniones sobre el tema. Respecto de este último objetivo, quiero ubicar el problema de la transparencia dentro de estas grandes temáticas y, por lo tanto, hacerla parte de una tradición más amplia, antigua y familiar. Y ello no con el fin de domesticar un tópico como la transparencia. Por el contrario, quiero sugerir que, a pesar de la tradición y la historia, la transparencia puede alzarse como el más grande e importante tópico mientras que la libertad de expresión y la de prensa -o, al menos, algunos aspectos de ellas- son solo una esquina históricamente destacada de una inquietud más grande, interesante y omnipresente.

${ }^{26}$ Véase McIntyre v. Ohio Elections Comm'n, 514 U.S. 334 (1995) (protegiendo el derecho a participar en discursos políticos anónimos); Talley v. California, 362 U.S. 60 (1960) (lo mismo); véase también Nat'l Ass'n for the Advancement of Colored People v. Alabama, 357 U.S. 449 (1958) (determinando un derecho de la Primera Enmienda a no divulgar el listado de miebros de una organización); Chesa Boudin, Note, Publius and the Petition: Doe v. Reed and the History of Anonymous Speech, 120 Yale L.J. 2140 (2011).

27 Véase Branzburg v. Hayes, 408 U.S. 665 (1972) (rechazando la pretensión de que el privilegio de un periodista estaba protegido por la cláusula de la prensa en la Primera Enmienda); In re Grand Jury Subpoena, 397 F.3d 964 (D.C. Cir. 2005) (lo mismo). Muchos estados han establecido tal privilegio por vía de la legislación, véase, por ejemplo, Ark. Code ANN. § 16-85-510 (2005); Neb. Rev. Stat. § 20-144 (2007), y la Free Flow of Information Act of 2007, H.R. 2102, 110th Cong. § 2 (2007), es, al momento de escribir esto, pendiente en el Congreso, aunque con decrecientes posibilidades de ser aprobada.

28 * Nota de los traductores: El autor hace referencia al adagio "where you stand depends on where you sit", que posee un juego de palabras que enfatiza la relatividad de todo juicio y de cómo este depende al final de la posición que tengamos al momento de emitirlo.

${ }^{29}$ Véase supra nota 10 para una muestra de textos recientes. 


\section{DEFINICIONES PRELIMINARES}

La transparencia es una metáfora, por supuesto; o quizás sea mejor entendida como una analogía. En su uso principal, la palabra "transparencia" es empleada para referir a una propiedad física. Ser transparente, nos dice el Oxford English Dictionary, es tener "la propiedad de transmitir luz, de manera tal de hacer completamente visibles a los cuerpos que se encuentran más allá” ${ }^{30}$. Al ser usada metafóricamente, por lo tanto, entendemos que ser transparente es la capacidad de ser observado sin distorsión. En consecuencia, el ser transparente para un hecho, información o proceso importa estar abierto y disponible para su examen y control.

Así las cosas, la transparencia es mejor entendida como un atributo pasivo o negativo. Transparencia versa sobre disponibilidad y accesibilidad, pero tales atributos de ella son agnósticos respecto de la pregunta sobre quién se beneficia de la disponibilidad y accesibilidad y a qué costo. En la clásica distinción de Isaiah Berlin entre libertad positiva y negativa ${ }^{31}$, se argumenta que es esencial distinguir la libertad de sus condiciones de ejercicio y, por tanto, distinguir libertad negativa respecto de lo que él engañosamente designó como libertad "positiva" 32 . En consecuencia, en tanto la transparencia es más bien un atributo que una actividad (como escribir o hablar) o un poder, calza correctamente al interior de la libertad negativa en la ahora familiar distinción entre libertad positiva y negativa o derechos positivos y negativos ${ }^{33}$. Leyes de reuniones abiertas, por ejemplo, sirven a las finalidades de la transparencia en tanto prohíben que ciertos órganos gubernamentales cierren determinados procedimientos a personas interesadas, pero la pregunta de si estas personas existen es un asunto completamente distinto ${ }^{34}$. Lo

\footnotetext{
302 The compact edition of the oxford english dictionary 3383 (1971).

31 Véase Isaiah Berlin, Two Concepts of Liberty, en Four essays on liberty 118 (1969).

${ }^{32}$ Un importante análisis y respuesta a Berlin se halla en Lawrence Crocker, positive liberty: an essay in normative political philosophy (1980).

33 Sobre derechos positivos y negativos, véase, por ejemplo, Larry A. Alexander, Constitutionalism, in The blackwell guide to the philosophy of law and legal theory 248, 255 (Martin P. Golding \& William A. Edmundson eds., 2005); Sotirios A. Barber, Welfare and the Instrumental Con-stitution, 42 AM. J. Juris. 159 (1997); Frank I. Michelman, Foreword: On Protecting the Poor Through the Fourteenth Amendment, 83 Harv. L. Rev. 7 (1969); Albie Sachs, Enforcement of Social and Eco-nomic Rights, 22 AM. U. INT'L L. Rev. 673 (2007); Ellen Wiles, Aspirational Principles or Enforceable Rights?: The Future for SocioEconomic Rights in National Law, 22 AM. U. INT'L L. Rev. 35 (2006). La antigua y duradera resistencia de los Estados Unidos a reconocer derechos positivos en el Derecho Constitucional se grafica, por ejemplo, en DeShaney v. Winnebago Cnty. Dep't. of Soc. Servs., 489 U.S. 189 (1989); San Antonio Indep. Sch. Dist. v. Rodriguez, 411 U.S. 1 (1973).

${ }^{34}$ En general, sobre leyes de reuniones abiertas véase el comprensivo y equilibrado tratamiento en Nicholas Johnson, Open Meetings and Closed Minds: Another Road to the Mountaintop, 53 Drake L. Rev. 11 (2004). También útil, por ejemplo, John F. O’Connor \& Michael J. Baratz, Some Assembly Required: The Application of State Open Meeting Laws to Email Correspondence, 12 GEO. Mason L. Rev. 719 (2004); Daxton R. "Chip" Stewart, Let the Sunshine in, or Else: An Examination of the "Teeth" of State and Federal Open Meetings and Open Records Laws, 15 COMM. L. \& POL'Y 265 (2010). Este asunto no es nuevo. Véase Note, Open Meeting Statutes: The Press Fights for the "Right to Know", 75 Harv. L. Rev. 1199 (1962). $\mathrm{Y}$ ahora está bien establecido que no existe un derecho positivo de acceso a reuniones y procedimientos
} 
mismo puede decirse de las leyes y decisiones de la Corte Suprema que exigen que los juicios sean abiertos al público ${ }^{35}$. Y también de las leyes de acceso a la información ${ }^{36}$, que obligan a que el gobierno deje a disposición determinados archivos y documentos solicitados por el público aunque no sean necesariamente pedidos por alguien. Una concepción positiva de la transparencia podría apoyar los esfuerzos tendientes a hacer que la información sea además fácilmente utilizable y no simplemente disponible. Tal es la diferencia, por ejemplo, entre una obligación de publicación de información y una solicitud de acceso. Sin embargo, la mayoría de las concepciones actuales sobre transparencia atienden más a la disponibilidad que a la accesibilidad de la información. Incluso más, sería casi impensable imaginar leyes que ordenen cómo debiese usarse o procesarse la información que ya se encuentra disponible gracias a las normas sobre transparencia. Como resultado, la transparencia es distinguible del conocimiento toda vez que ella puede fomentarlo, y es, en el mejor de los casos, un facilitador de él no pudiendo ser confundida con el conocimiento mismo.

La transparencia puede facilitar el conocimiento, pero su capacidad actual de hacerlo depende de numerosos factores, entre ellos el grado de transparencia misma. Es común que las personas describan procesos como transparentes o no transparentes, pero la transparencia es de hecho variable. El lenguaje ordinario distingue transparencia de translucidez y, por tanto, reconoce la existencia de una transparencia parcial. Este último término, sin embargo, no ha penetrado todavía en el discurso político o jurídico contemporáneo. Pese a ello, el mismo hecho que distingamos transparencia -o transparencia parcial- de translucidez demuestra que, en ocasiones, es valioso reconocer distintos grados de transparencia. Documentos redactados a base de la seguridad nacional ${ }^{37}$, por ejemplo, son menos susceptibles de ser por completo transparentes aunque tampoco totalmente opacos. Las leyes de reuniones abiertas establecen que los procedimientos de los concejos comunales, las juntas educacionales y las agencias administrativas sean públicos, aunque varias de ellas permiten que algunas discusiones se den a puertas cerradas, literal o figurativamente. Bajo esta normativa, sin embargo, se ha generado

gubernamentales, ya sea para la prensa o para el público en general. Véase Houchins v. KQED, Inc., 438 U.S. 1 (1978); Pell v. Procunier, 417 U.S. 817 (1974); Saxbe v. Wash. Post Co., 417 U.S. 843 (1974); cfr. Madison Joint Sch. Dist. No. 8 v. Wis. Emp't Relations Comm'n, 429 U.S. 167 (1976). Para un argumento en contra, véase Adam M. Samaha, Government Secrets, Constitutional Law, and Platforms for Judicial Intervention, 53 UCLA L. REV. 909 (2006).

35 Richmond Newspapers, Inc. v. Virginia, 448 U.S. 555 (1980). Para una elaboración posterior, véase Press-Enter. Co. v. Superior Court, 478 U.S. 1 (1986); Press-Enter. Co. v. Superior Court, 464 U.S. 501 (1984); Seattle Times Co. v. Rhinehart, 467 U.S. 20 (1984); Globe Newspaper Co. v. Superior Court, 457 U.S. 596 (1982).

36 Acta de Acceso a Información (Freedom of Information Act de 1986), 5 U.S.C. $\$ 552$ (2006). Para una compilación de leyes estatales de acceso a la información, véase State FOI Laws, Nat'l Freedom Info. Coalition, http://www.nfoic.org/state-foi-laws (last visited May 16, 2011).

37 Véase Classified Information Procedures Act, Pub. L. No. 96-456, § 6(c), 94 Stat. 2025, 2027 (1980); Thomas Grexa, Title VII Tenure Litigation in the Academy and Academic Freedom-A Current Appraisal, 96 Dick. L. Rev. 11, 32 n.168 (1991); Jonathan H. Marks, 9/11 +3/11 + 7/7 = ?: What Counts in Counterterrorism, 37 Colum. Hum. RTS. L. Rev. 559, 617 n. 255 (2006) 
un bajo volumen de litigación acerca de las transacciones y discusiones que se califican como sesiones para efectos de las leyes de reuniones abiertas y las que se encuentran bajo aquella serie de exenciones y excepciones a esa transparencia ${ }^{38}$. Pero la existencia de tal litigación no debería restar validez del fenómeno mayor. Un ejército de abogados y académicos se ganan la vida litigando o analizando las excepciones al Acta de Acceso a la Información ${ }^{39}$. Pese a ello sería difícil negar que tras la aprobación de dicha Acta, Estados Unidos es un lugar diferente al de antes de su promulgación.

Además de la existencia de distintos grados de transparencia que se manifiestan en qué archivos, procedimientos o información están o no disponibles, sus grados pueden también variar en relación con el tamaño e identidad de la audiencia. La asistencia a las reuniones de facultad de mi institución, por ejemplo, está limitada a los miembros de la facultad y por tanto cerradas para estudiantes, exalumnos, abogados que no son parte de la facultad y público en general. Como resultado de esto, los procedimientos carecen de transparencia para un número de partes interesadas. Por el contrario, el hecho de que estos procedimientos estén abiertos para todos los miembros de la facultad y no solo para un subgrupo de ellos, los hace más transparentes que muchas otras alternativas imaginables. De igual modo, no cualquier ciudadano está habilitado para transitar por los salones de la Casa Blanca y volar en aeronaves militares ${ }^{40}$. Solo los periodistas acreditados pueden hacerlo. Esto genera una situación que podría ser descrita, en términos más precisos, como de transparencia parcial. Por otra parte, cada vez que un documento está disponible solo a quienes están incluidos en la etiqueta “para su conocimiento”, nos encontramos en una situación que se ubica en algún punto entre la total transparencia y la total opacidad.

En cualquier discusión sobre transparencia, por tanto, es vital reconocer que la transparencia es una relación de tres partes o, de otra forma dicho, una relación de tres variables diferentes. Cualquier indagación sobre transparencia presentará, primero, la pregunta sobre qué persona o institución se involucra en los procedimientos o posee documentos o información; segundo, la pregunta sobre qué actividades, procedimientos, información o documentos deben ser transparentables; y, tercero, cuál es el tipo de individuos o de instituciones que están legitimados para acceder a dichas actividades, procedimientos, información o documentos. De esta forma, sin especificar quién debe

${ }^{38}$ Véase, $v$. gr., Stephen Schaeffer, Comment, Sunshine in Cyberspace: Electronic Deliberation and the Reach of Open Meeting Laws, 48 ST. Louis U. L.J. 755 (2004); Timothy P. Whelan, New York's Open Meetings Law: Revision of the Political Caucus Exemption and Its Implications for Local Government, 60 Brook. L. Rev. 1483 (1995).

${ }^{39}$ Véase, $v$. gr. U.S. Dep't of Just., Freedom of information act reference guide (2010), available at http:// www.justice.gov/oip/referenceguide.htm.

40 Por cierto existen muchos problemas importantes sobre quién está incluido o excluido en un sistema de acceso parcial, véase Nation Magazine v. U.S. Dep't of Def., 762 F. Supp. 1558 (S.D.N.Y. 1991) (rechazando un cuestionamiento al procedimiento y criterio del Departamento de Defensa para determinar qué periodistas serán miembros de los “pools de prensa” en tiempo de guerra); Frederick Schauer, Parsing the Pentagon Papers (May 1991) (paper no publicado, Joan Shorenstein Center, Harvard University, John F. Kennedy School of Government). Sin perjuicio de ello, tales problemas no son mi principal preocupación aquí. 
hacer disponible qué para quiénes, es poco probable que podamos entender el tipo de transparencia respecto del cual estamos discutiendo.

\section{LOS OBJETIVOS DE LA TRANSPARENCIA}

Luego de las definiciones preliminares nos abocaremos ahora a las supuestas virtudes de la transparencia. Preferentemente entre estas virtudes -al menos según nos indica gran parte del discurso contemporáneo- está la que comúnmente se describe como "rendición de cuentas" (accountability). En efecto, muchos argumentan que la transparencia de una institución con otra, o la transparencia de una institución con el público, aumenta la rendición de cuentas (accountability) de la institución transparente ${ }^{41}$. Sin embargo, la frase "rendición de cuentas" (accountability) incluye presuntas virtudes como permitir a una institución ser controlada por otra y también la participación pública por sí misma. Por ello, quiero dejar de lado la frase "rendición de cuentas" y distinguir entre transparencia como regulación y transparencia como democracia o participación. En términos amplios incluso, la transparencia está pensada para servir a cuatro valores que quisiera diferenciar claramente. Diferenciaré, entonces, entre Transparencia como Regulación, Transparencia como Democracia, Transparencia como Eficiencia y Transparencia como Epistemología.

\section{A. Transparencia como regulación}

Si nos enfocamos primero en lo que toca a la transparencia como regulación, debemos recordar aquel antiguo adagio que nos dice que "información es poder" 42 . En este sentido, uno de los aspectos de la transparencia es la constatación de que cuando una persona o institución tiene información respecto de otra ello refleja que la primera tiene poder sobre la segunda. Cómo se ejerce exactamente ese poder variará considerablemente dependiendo del contexto. Consideremos, por ejemplo, la extorsión. El extorsionador tiene información -o conocimiento- sobre la víctima y ese poder sobre ella deriva precisamente de la existencia de información que la víctima desea mantener en secreto. De esta forma, la transparencia que maneja el extorsionador -transparencia, ciertamente, no facilitada por la víctima y socialmente indeseable ${ }^{43}-$ le permite

${ }^{41}$ En efecto, es común usar conjuntamente las palabras "transparente" (o "transparencia”) y "accountable" (o accountability, en tanto "rendición de cuentas"). Véase, v. gr., Blakely v. Washington, 542 U.S. 296,316 (2004).

${ }^{42}$ Y la pertinente "información es poder" deriva de la expresión de Francis Bacon, "conocimiento es poder", que es la traducción común de nam et ipsa scientia potestas est. John Bartlett, Familiar Quotations 111 (Christopher Morley \& Louella D. Everett eds., 12th ed. 1950) (citando a Francis Bacon, Haeresibus, in Meditaciones Sacrae (1597)).

43 El empleo del "casi" en el texto es intencional, reflejando un antiguo debate sobre extorsiones - la amenaza de publicar información correcta bajo circunstancias en las que la divulgación misma sería completamente legal-y si la criminalización se justifica en caso alguno. Entre las más prominentes incursiones 
controlar a la víctima, lo que es, en definitiva, el objetivo primordial de la extorsión. La extorsión puede ser socialmente indeseable pero no obstante nos brinda un buen ejemplo sobre la forma en la que la posesión de información sobre otro típicamente facilita el poder o control sobre este.

Más importante para el tópico general de la transparencia es el amplio rango de contextos en los que ella se comporta como una forma en que dicha institución puede ser controlada o regulada por otra. La divulgación obligatoria que pesa sobre sociedades respecto de numerosos reportes financieros y transacciones es una importante dimensión de regulación corporativa y de valores, dimensión esta última que se encuentra basada en la premisa de que una sociedad en la que las finanzas, la organización interna y los principales contratos son accesibles para el público y sus accionistas es una sociedad que se encuentra limitada en su habilidad para involucrarse en una serie de prácticas que dañen a los inversionistas ${ }^{44}$.

En términos similares, la transparencia de las tasas de mortalidad en los hospitales está pensada para fomentar mejor el cuidado sanitario ${ }^{45}$. La transparencia de la violencia en los campus universitarios se ha esgrimido para aumentar la preocupación por seguridad e incrementar la capacidad de los estudiantes y apoderados para adoptar decisiones sabias sobre dónde deberían educarse los estudiantes ${ }^{46}$. Por su parte, la transparencia de las inspecciones de higiene en restaurantes aparentemente provee un incentivo para los dueños de ellos de velar que sus establecimientos estén limpios y saludables ${ }^{47}$. En estas y en otras numerosas instancias, publicidad y transparencia están pensadas como un importante componente de una amplia estrategia regulatoria. De hecho, cuando Cass

del debate se encuentran Ronald H. Coase, Lecture, Blackmail, 74 VA. L. Rev. 655 (1988); Richard A. Epstein, Blackmail, Inc., 50 U. CHI. L. REV. 553 (1983); Henry E. Smith, The Harm in Blackmail, 92 NW. U.L. Rev. 861 (1998); Symposium, Blackmail and Other Forms of Arm-Twisting, 141 U. PA. L. Rev. 1567 (1993). A valuable recent recapitulation and analysis is Ken Levy, The Solu- tion to the Real Blackmail Paradox: The Common Link Between Blackmail and Other Criminal Threats, 39 Conn. L. Rev. 1051 (2007).

${ }^{44}$ Véase Allen Ferrell, The Case for Mandatory Disclosure in Securities Regulation Around the World, 2 Brook. J. Corp. Fin. \& Com. L. 81 (2007); Edward Rock, Securities Regulation As Lobster Trap: A Credible Commitment Theory of Mandatory Disclosure, 23 Cardozo L. Rev. 675 (2002); Gary F. Goldring, Note, Mandatory Disclosure of Corporate Projections and the Goals of Securities Regulation, 81 Colum. L. Rev. 1525 (1981). Para una mirada escéptica sobre las supuestas ventajas de la publicidad en generar un comportamiento sustantivamente deseable, véase Ripken, supra note 19. Diferentes formas de escepticismo también se encuentran en Carol J. Simon, The Effect of the 1933 Securities Act on Investor Information and the Performance of New Issues, 79 AM. ECON. Rev. 295 (1989).

${ }^{45}$ Véase Clark C. Havighurst, Regulations of Health Facilities and Services by "Certificate of Need", 59 VA. L. Rev. 1143, 1163 n. 76 (1973); Michael B. Rothberg et al., Choosing the Best Hospital: The Limitations of Public Quality Reporting, 27 health AFF. 1680 (2008); Clayton Christensen \& Jason Hwang, What Obama's Health Care Team Can Learn from Massachusetts, harv. Bus. Rev. Blog (Jan. 22, 2009, 5:23 PM), http://blogs.hbr.org/hbr/on-innovation/2009/01/what-obamas-health- care-team-c.html.

46 Véase Jeanne Clery Disclosure of Campus Security Policy and Campus Crime Statistics Act, 20 U.S.C. § 1092(f) (2006); Oren R. Griffin, Constructing a Legal and Managerial Paradigm Applicable to the Modern-Day Safety and Security Challenge at Colleges and Universities, 54 ST. Louis U. L.J. 241 (2009).

47 Véase en general Ginger Zhe Jin \& Phillip Leslie, The Effect of Information on Product Quality: Evidence from Restaurant Hygiene Grade Cards, 118 Q. J. Econ. 409 (2003). 
Sunstein y Richard Thaler escribieron un artículo llamado "La Publicidad es la Mejor Forma de Regulación del Crédito" 48 y cuando Gordon Crovitz escribió en el Wall Street Journal que la "Transparencia es más Poderosa que la Regulación” ${ }^{49}$, ellos reconocieron explícitamente que requerir de entidades reguladas que desarrollen sus actividades en forma abierta respecto de aquellos que buscan ejercer control sobre esas entidades es en sí misma una forma de regulación. Y ello aun cuando se mantenga como pregunta abierta si la transparencia como regulación es mejor o peor -luego de considerar todas las variables- que otras formas directas de regulación ${ }^{50}$. Sin perjuicio de ello, una vez que consideramos que la transparencia puede ser una forma de control -y por tanto una forma de regulación- podemos observar que en muchas de las discusiones sobre transparencia, no es un error preguntarse si es bueno que quienes son obligados o se esperan que sean transparentes, deban o no estar sujetos a dicho control.

\section{B. Transparencia como democracia}

Aunque los debates sobre transparencia como estrategia regulatoria son cada día más comunes, existe un determinado tipo de control o regulación que domina muchas de las discusiones sobre transparencia, y es la que ejerce el pueblo o sus representados elegidos (o autonombrados) sobre el gobierno. En búsqueda de una mejor palabra, podemos simplemente llamar al control público de gobernantes y gobierno como "democracia". En la medida que la transparencia de los procesos de gobierno hacia el pueblo es promovida como una forma de facilitar el control público sobre el gobierno y sus decisiones ${ }^{51}$, se puede entender este principio como uno de Transparencia como Democracia.

Sin embargo, la transparencia como facilitadora de la democracia y como vehículo para que los gobernados controlen a los gobernantes tiene dos importante dimensiones distintivas. Primero, la transparencia puede, tal como se afirma, reducir la corrupción, los sobornos, la captura regulatoria y otras formas de conductas gubernamentales indebidas y, en ese sentido, la transparencia gubernamental es simplemente una forma de transparencia como regulación ${ }^{52}$. Esto es lo que presumiblemente quería decir el juez Brandeis al proclamar célebremente que la luz del sol era el mejor de los desinfectantes ${ }^{53}$. Y ello, aunque la democracia no implique necesariamente que el pueblo sea mejor que los

48 Thaler \& Sunstein, supra note 15.

${ }^{49}$ Crovitz, supra note 15 .

50 Véase Ripken, supra note 19.

51 Véase supra note 12.

52 En efecto, la apreciación sobre la transparencia como la mejor forma de prevenir la corrupción es el principio guía de Transparency International, supra note 21, y del ímpetu detrás del ampliamente usado Índice de Percepción de la Corrupción de Transparency International. Véase Corruption Perceptions Index 2010 Results, Trans-Parency Int'l, http://www.transparency.org/policy_research/surveys_indices/cpi/2010/ results (last visited May 16, 2011).

53 "La publicidad es justamente recomendada como un remedio para enfermedades sociales e industriales. Se dice que la luz del sol es uno de los mejores desinfectantes; la luz eléctrica, uno de los policías más eficientes". Louis D. Brandeis, Other people's money and bow the bankers Use It 92 (1914). 
funcionarios públicos remunerados en la toma de decisiones ${ }^{54}$. La segunda dimensión de la Transparencia como Democracia hace referencia al control público. Y ello no por razones de facilitar mejores decisiones, sino que, en cambio, como la encarnación del control público como un fin en sí mismo. La democracia, después de todo, no hace referencia a que las personas estén en lo correcto, sino que al derecho del pueblo a equivocarse. Desde esta perspectiva, las leyes de reuniones abiertas, las de acceso a información pública y muchos de los otros dispositivos de transparencia difícilmente son, para la frustración de aquellos cuyas actividades y registros deben ser transparentes, una garantía fiable de toma de decisiones sabias. No obstante, estos dispositivos de publicidad y transparencia son elementos facilitadores de la toma de decisión pública que constituyen, en ocasiones para mejor y en otras para peor, un importante componente del gobierno democrático.

Así las cosas, es difícil negar que las decisiones transparentes a veces son peores simplemente por su transparencia. El juez Souter, que se retiró de la Corte Suprema por razones distintas que la inminente posibilidad de televisar los argumentos ante dicha Corte, célebremente apuntó que "el día en que se vea una cámara [de televisión] ingresar a la sala de la Corte, tendrá que ser sobre mi cadáver" 55 . Para el juez Souter y muchos otros, el creciente involucramiento del público y la inspección de ciertos procedimientos de toma de decisiones opera, más bien, como una suerte de Ley de Gresham o como populismo en su sentido peyorativo ${ }^{56}$, creando un ambiente decisional en el que el mínimo común denominador de involucramiento generalizado del público causaría que los buenos argumentos sean desplazados por los malos.

\section{Transparencia como Eficiencia y Transparencia como Epistemología}

La visión de aquellos que afirman que la transparencia favorece procesos y resultados subóptimos es discutible y debe contextualizarse. Aquellos que reconocen que la información abierta es clave para los mercados eficientes, esto es, aquellos que postulan la Transparencia como Eficiencia, están argumentando que la libre disponibilidad de información es lo que precisamente permite la operación eficiente de los mercados $^{57}$. En efecto, esta reflexión no es exclusiva de los mercados en sentido convencional. La familiar

${ }^{54}$ Para el contraste entre justificaciones epistémicas y alternativas de la democracia, véase en general William N. Nelson, on justifying democracy (1980).

55 On Cameras in Supreme Court, Souter Says, 'Over My Dead Body', N.Y. Times, Mar. 30, 1996, at 24 (citando al juez Souter). Sobre el tema en general, incluyendo las diversas opinions de los otros jueces, véase Robert L. Brown, Just a Matter of Time? Video Cameras at the United States Supreme Court and the State Supreme Courts, 9 J. APP. Prac. \& Process 1 (2007).

${ }^{56}$ En contra de la opinión que el populismo es justamente democrático cuando causa resultados con los que algún comentarista usando el término discrepa, véase John Lukacs, democracy and populism: fear and batred 5-8 (2005).

57 Véase Allison Stanger, one nation under contract: the outsourcing of American power and the future of foreign policy 178-79 (2009); Richard Dolinar \& S. Luke Leininger, Pay for Performance or Compliance? A Second Opinion on Medicare Reimbursement, 3 Ind. Health L. Rev. 397, 418-20 (2006). 
metáfora del mercado de las ideas en la teoría de la libertad de expresión ${ }^{58}$ es una variante de la idea de Transparencia como Eficiencia, que podríamos llamar Transparencia como Epistemología. Esta última comprendería la afirmación de que la abierta disponibilidad de información facilita la identificación de lo verdadero (y falso) y, consecuentemente, produce más conocimiento y mayor progreso. John Milton pensó más o menos esto en el siglo XVII ${ }^{59}$, John Stuart Mill reflexionó lo mismo en el siglo XIX ${ }^{60}$, Oliver Wendell Holmes hizo lo suyo en los primeros años del siglo $\mathrm{XX}^{61}$ y muchos en la comunidad del software libre (o de código abierto) ahora piensan algo bastante similar ${ }^{62}$. La libre disponibilidad de información -se sostiene- tiende hacia el conocimiento aunque no lo garantiza y, por ello, la transparencia es un camino altamente deseable y necesario en la ruta hacia la verdad.

Sin embargo, como se reconoce cada vez más en la literatura, algunas de las demandas por mayor transparencia o son optimistas o son exageradas ${ }^{63}$. Y algunas de ellas, en determinados contextos, pueden ser lisa y llanamente falsas. En efecto, así como la petición de transparencia acerca del lugar de nacimiento del presidente Obama o la demanda por los datos empíricos en que se basa el horóscopo no han producido efectos en la aceptación de estas premisas ${ }^{64}$, así como el juez Souter afirmaba su preocupación de que mayor luz solar sobre la Corte Suprema produciría peores argumentos y decisiones $^{65}$, así también ahora somos más escépticos sobre la correlación entre apertura y conocimiento $^{66}$ o entre la disponibilidad de la información y la calidad de las decisiones que se basan en ella.

Dejaré estas preguntas de lado, por el momento, pues mi objetivo -al menos en esta parte de la Conferencia- ha sido simplemente distinguir entre Transparencia

${ }^{58}$ Véase Frederick Schauer, free speech: a philosophical enquiry 15-17 (1982); véase también Alvin I. Goldman \& James C. Cox, Speech, Truth, and the Free Market for Ideas, 2 Legal Theory 1, 2-3 (1996); Stanley Ingber, The Marketplace of Ideas: A Legitimizing Myth, 1984 duke L.J. 1, 2-3; William P. Marshall, In Defense of the Search for Truth As a First Amendment Justification, 30 GA. L. Rev. 1, 1 (1995).

59 “¿Quién ha conocido caso en que la Verdad haya sido perjudicada en un encuentro libre y abierto?”. Milton's Areopagitica: a speech for the liberty of unlicensed printing 45 (H.B. Cotterill ed., Macmillan 1959) (1644).

${ }^{60} \mathrm{John}$ Stuart Mill, on liberty 18-24 (David Spitz ed., W.W. Norton \& Co. Inc. 1975) (1859).

61 "[L]a mejor prueba de veracidad es el poder de [una proposición de] ser aceptada en sí misma a través de la competencia del mercado..." Abrams v. United States, 250 U.S. 616, 630 (1919) (Holmes, J., dissenting); véase también Vincent Blasi, Holmes and the Marketplace of Ideas, 2004 SUP. CT. Rev. 1, 1-2, 4.

62 "Código abierto es un método de desarrollo de software que aprovecha el poder de distribución de revisión de pares y la transparencia del proceso. La promesa del código abierto es mejor calidad, mayor fidelidad, más flexibilidad, bajos costos y el fin de los amarres depredadores de un único proveedor". Mission, Open Source Initiative, http://www.opensource.org (last visited May 16, 2011).

${ }^{63}$ Véase Goldman \& Cox, supra note 57, at 29-32; Ingber, supra note 57, at 16-49; Frederick Schauer, Facts and the First Amendment, 57 UCLA L. Rev. 897, 908-12 (2010); Frederick Schauer, Is It Better to Be Safe Than Sorry?: Free Speech and the Precautionary Principle, 36 PEPP. L. Rev. 301, 308-12 (2009).

${ }^{64}$ Sobre la Primera Enmienda y las proposiciones fácticas y científicas, véase Schauer, Facts and the First Amendment, supra note 62.

65 Véase supra note 54.

${ }^{66} \mathrm{O}$ al menos deberíamos serlo. 
como Regulación, Transparencia como Democracia, Transparencia como Eficiencia y Transparencia como Epistemología, recordando que no podemos evaluar las medidas de transparencia en uno u otro espacio de toma de decisiones sin primero comprender cuáles son las ventajas que la transparencia supuestamente debe generar.

\section{EL CONSERVADURISMO DE LA TRANSPARENCIA}

Una vez que entendemos que la transparencia de una persona es el populismo de la otra y que comprendemos la ambigua reputación del populismo, entonces tenemos una base útil para pensar acerca de la transparencia como dispositivo de diseño institucional en la toma de decisiones, cuyas ventajas y desventajas, vicios y virtudes, costos y beneficios, pueden ser evaluados con las herramientas de la moderna teoría de decisiones. En efecto, para cualquier toma de decisión podemos prever la posibilidad de cometer dos tipos de errores: uno es el resultado de una mala decisión y el otro es el que impide adoptar una decisión correcta. Algunos reconocerán este marco en los términos de la distinción de los estadísticos entre errores Tipo I y Tipo II, mientras que otros estarán inclinados a usar la terminología de falsos positivos y falsos negativos. Si me quedo en cama todo el día es poco probable que me atropelle un automóvil, pero también es poco probable que coseche los beneficios de interactuar con otros. Tal como el derecho de las medidas cautelares establece $-y$, más precisamente, la regla del daño irreparable-, la decisión sobre una medida cautelar puede fallar cuando esta no es concedida a un demandante que está, de hecho, en lo correcto y cuya demanda eventualmente se acogería en el fondo, pero también puede fallar cuando se concede una medida cautelar a un actor cuya demanda carece de fundamento razonable y que posteriormente puede perder tras la conclusión del procedimiento ${ }^{67}$. Este análisis suele ser descrito en términos de "ponderación de valores" (balance of equities) o "ponderación de penurias"68 (balance of hardships) y la idea básica que hay en él es que el análisis que conduce a la decisión debe ponderar entre los daños probables de una acción errónea y los daños probables de una omisión errónea, todo ello bajo las condiciones de incertidumbre existentes al momento de adoptar la medida cautelar, esto es, antes de que se desarrolle por completo el procedimiento y de que se dé una oportunidad completa para desarrollar todos los hechos relevantes del caso.

La transparencia es similar. Transparencia Internacional es una organización dedicada particularmente a prevenir la corrupción y, en su concepto, procedimientos gubernamentales abiertos -reuniones abiertas, leyes de acceso a la información, libertad de prensa, publicidad del estado financiero de autoridades públicas y otros agentes- harían que la corrupción, los sobornos y otros ejemplos similares fuesen de más difícil ocurrencia. En

${ }^{67}$ Véase Owen M. Fiss, injunctions 9 (1972); Douglas Laycock, the death of the irreparable injury rule 8-11 (1991).

${ }^{68}$ Véase, v. gr., Metro. Taxicab Bd. of Trade v. New York, 615 F.3d 152, 156 (2d Cir. 2010); Small v. Operative Plasterers' and Cement Masons' Int'l Ass'n Local 200, 611 F.3d 483, 489-90 (9th Cir. 2010); Hanselman v. Frank, 77 Mass. App. Ct. 1104 (Mass. App. Ct. 2010). 
otros términos, la transparencia haría más difícil a los malos funcionarios involucrarse en las actividades de beneficio privado que comúnmente denominamos corrupción. Tal como lo afirmó Jeremy Bentham hace varias generaciones: "mientras más estrictamente vigilamos, mejor nos comportamos" ${ }^{69}$. Sin embargo, no debemos olvidar el comentario del juez Souter o los esfuerzos de la ahora Secretaria de Estado, antes Primera Dama, Hillary Clinton, para mantener negociaciones cerradas sobre el sistema de salud con las partes interesadas -el llamado Grupo de Trabajo para la Reforma Nacional del Sistema de Salud-a comienzos de los $1990^{70}$. La transparencia bien puede disuadir a los malos empleados públicos involucrarse en actos de corrupción u otras malas prácticas pero también el juez Souter, la secretaria Clinton y múltiples otros han reconocido que la transparencia puede hacer más difícil tomar decisiones correctas para buenos empleados públicos. Solo pregunte a cualquier Presidente, juez, o decano de Facultad de Derecho. Si esta conclusión es correcta -que la transparencia puede también ser un impedimento para la sabia toma de decisiones de los operadores ${ }^{71}$, entonces debemos revisar nuevamente los dos tipos de posibles errores. Uno es el error y consiguiente daño que se deriva de fallar en prevenir empleados públicos corruptos y sus malas prácticas. El otro es el error y consiguiente daño que emana de impedir que empleados públicos probos adopten sabias decisiones y ejecuten actividades potencialmente beneficiosas.

El diseño de cualquier escenario de toma de decisiones debe necesariamente resolver el problema de la probabilidad comparativa del daño (daño esperado) entre ambos tipos de errores. Cuando Blackstone observó que "es mejor que diez personas culpables escapen a que un inocente sufra" 72 , desarrollaba este tipo de análisis incluso antes que

${ }^{69}$ Jeremy Bentham, Farming Defended, in 1 Writings on the poor laws 276, 277 (Michael Quinn ed., Oxford University Press 2001) (1796).

${ }^{70}$ Véase Ass'n of Am. Physicians \& Surgeons, Inc. v. Clinton, 813 F. Supp. 82 (D.D.C. 1993), rev'd 997 F.2d 898 (D.C. Cir. 1993) (abordando preguntas constitucionales y legales sobre si los asesores directos del Presidente pueden ser obligados por ley a mantener sus deliberaciones públicamente). Sobre el tema constitucional, véase Pub. Citizen v. U.S. Dep't of Justice, 491 U.S. 440, 482-89 (1989) (Kennedy, J., concurring); Jay S. Bybee, Advising the President: Separation of Powers and the Federal Advi-sory Commission Act, 104 Yale L.J. 51 (1994).

${ }^{71} \mathrm{El}$ por qué es nuevamente una función en la pregunta sobre la relación entre conciencia y verdad (o congruencia). Si la veracidad o congruencia de una proposición tiene un considerable poder explicativo en la determinación de las proposiciones que serán aceptadas y rechazadas por algún sector, entonces aumenta la conciencia sobre proposiciones verdaderas y de hechos descritos con precisión -el objetivo teórico principal de un régimen de la transparencia- aumentará el grado de aceptación de tales proposiciones y, por tanto, incrementará el conocimiento. Pero si, tal como aparece creciente e inquietantemente en muchas áreas de la vida pública, la verdad tiene una conexión causal limitada con la aceptación, entonces la transparencia puede ser en ocasiones contraproductiva. Un adagio bien conocido entre abogados apelantes es que debes dejar a tu cliente en casa durante un alegato de apelación, precisamente, porque el tipo de argumentos que apelan a los clientes son difícilmente persuasivos a los jueces de apelación. Y mientras que la transparencia tiene este mismo problema, esta puede a veces empoderar argumentos débiles y hacer que los argumentos más sólidos o razonables tengan más dificultad de avanzar.

7215 William Blackstone, commentaries $* 434$. Para un reciente y relevante análisis, véase Alexander Volokh, n Guilty Men, 146 U. PA. L. Rev. 173 (1997). 
Howard Raiffa ${ }^{73}$, quien ha sido quizás el principal exponente de esta idea en la era de la moderna teoría de decisiones.

Si aplicamos, en términos generales, las lecciones de la teoría de las decisiones a la pregunta de la transparencia o, más específicamente, si asumimos que una toma de decisión transparente y, por tanto, más abierta y populista es subóptima para un gran número de decisiones que podrían adoptarse por sabios y bienintencionados tomadores de decisiones, entonces podemos observar el conservadurismo inherente -en el sentido no político del término- del llamado de la transparencia. En efecto, el reclamo por mayor transparencia puede ser entendido como una manifestación de la creencia de que el error en la prevención frente a la corrupción o frente a tomadores de decisiones insensatos que adoptan medidas dañosas es un error mucho más serio que el error de disuadir a los sabios y a los tomadores de decisiones no corruptos de adoptar medidas que pueden adoptarse de una forma más sencilla en la oscuridad que a la luz del sol.

De esta forma, al buscar evitar malas decisiones al costo de evitar algunas buenas, la transparencia puede ser vista como comprometida con la creencia de que es mejor impedir un número de malas decisiones incluso asumiendo el costo de impedir algunas buenas, más que maximizar el número de buenas decisiones asumiendo incluso el costo de aceptar un mayor número de las malas. De esta forma, al acometer exactamente esa estrategia -cortar tanto el extremo derecho como el izquierdo de la distribución de probabilidad- la transparencia es entendida como una aproximación conservadora al diseño de instituciones. En algunos momentos y lugares, tal conservadurismo está bien justificado. Si fuese un zimbabuense, me preocuparía considerablemente de las malas decisiones adoptadas lejos de la mirada de la población y de la comunidad internacional y, por el contrario, no me preocuparía demasiado de impedir las buenas decisiones del gobierno de Robert Mugabe. En este caso, la transparencia probablemente evitaría algunas malas decisiones pero no impediría muchas buenas decisiones (o incluso una buena decisión). En otros momentos y lugares, sin embargo, el conservadurismo de la transparencia gana poco y genera un alto costo. El juez Souter tenía una intuición correcta, en virtud de ello, sin perjuicio de que puedan existir considerables beneficios educacionales para el público al televisar los argumentos orales ante la Corte Suprema, los que son mayoritariamente ortogonales a mi argumento principal ${ }^{74}$, la Corte $-\mathrm{y}$, ciertamente, sus acuerdos e incluso en el caso más debatible de los argumentos orales-constituye un claro ejemplo de un procedimiento en que las desventajas de la transparencia pueden aplastar los beneficios. La observación de Bentham de que "mientras más estrictamente vigilamos, mejor nos comportamos” es correcta solo si definimos buen comportamiento como la ausencia de una mala conducta, más que la presencia de una conducta correcta. Es probablemente cierto que mientras exista una mayor y más estricta vigilancia, será menos probable que nos comportemos incorrectamente. Pero también es cierto que, en

${ }^{73}$ Howard Raiffa, decision analysis: introductory lectures on choices under uncertainty (1968); véase también R. Duncan Luce E Howard Raiffa, games and decisions: introduction and critical survey (1957).

${ }^{74}$ Esto es, que pueden existir ventajas en tener al público aprendiendo sobre la Corte Suprema, lo que es y lo que hace, incluso si este aprendizaje se desarrolla con el costo de un número mayor de decisiones erróneas. 
ocasiones, mientras más cerca somos observados, menos probable será que nos comportemos de manera admirable.

\section{LA OMNIPRESENCIA DE LA TRANSPARENCIA}

Para alguien que pasa parte de su tiempo como estudiante de la doctrina y teoría de la libertad de expresión, las discusiones sobre la transparencia contienen una intriga especial. En el artificial mundo de las Facultades de Derecho en el que habito, la libertad de expresión es un tópico para cursos y especialistas en Derecho Constitucional, mientras que el acceso a la información pública está ubicado en el Derecho Administrativo. Preguntas sobre software libre son de aficionados a la propiedad intelectual, leyes de reuniones abiertas son analizadas por aquellos que se concentran en derecho de gobiernos locales y estatales, y las cuestiones sobre divulgaciones obligatorias se distribuyen entre académicos que enseñan y escriben en las áreas de regulación del mercado de valores, sistema de salud o protección de consumidores. Estoy seguro que hay áreas que he olvidado mencionar en las que también las preguntas sobre publicidad y transparencia tienen un lugar significativo. Pero a pesar de toda la divergencia en las mallas curriculares de las Facultades de Derecho y de la doctrina jurídica, todos estos tópicos tratan sobre apertura, sobre disponibilidad, sobre acceso a ideas e información y, especialmente, sobre los impedimentos oficiales para el libre uso y transferencia de dichas ideas e información. En ese sentido, es extraño que tópicos tan claramente interrelacionados estén típicamente separados entre $s^{75}$.

Lo que es especialmente raro, sin embargo, es que esta compartida aproximación sobre información y apertura que desarrollamos entre áreas de conocimiento aparentemente diversas es extraña e infrecuente en mi aun más pequeña esquina del mundo -ese mundo habitado por vuestras tres Baum Lecturers de este año ${ }^{76}$, , ese mundo de quienes estudiamos la libertad de expresión y la libertad de prensa. En ese pequeño mundo -mi pequeño mundo- nos preocupamos incesantemente de las restricciones estatales a lo que oradores, escritores, editores y operadores de radio y televisión quieren decir, pero raramente nos preocupamos acerca de la peculiaridad de depender en tal grado en lo que tales individuos pueden querer decir, en contraposición al completo rango de medidas que pudieren fomentar un mayor número de discursos ${ }^{77}$. Si los argumentos epistémicos, informativos y democráticos a favor de la libertad de expresión son correctos, entonces la

75 Algunos de estos tópicos típicamente diversos son útilmente agrupados en James Boyle, Shamans, software, and spleens: Law and the construction of the information society (1996).

${ }^{76}$ Lee C. Bollinger, Baum Lecture 2010, 2011 U. ILL. L. Rev. 1011; Geoffrey R. Stone, Baum Lecture 2011, 2012 U. ILL L. Rev. (próximamente en 2012).

${ }^{77}$ Para mis observaciones preliminares sobre el fenómeno, véase en general Frederick Schauer, Hohfeld's First Amendment, 76 GEO. Wash. L. Rev. 914 (2008). La forma en que los valores que subyacen a la Primera Enmienda pudieren abogar por políticas positivas en adición a derechos negativos fue antes explorado en Steven H. Shiffrin, dissent, injustice, and the meanings of America (1999). 
ventaja que la libertad de expresión acarrea para la verdad, el conocimiento y la toma de decisiones públicas constituye, en ciertos contextos, una condición necesaria aunque no es ni por cerca una condición suficiente. Dicho de otra manera, y de manera más relevante para el tema de esta Conferencia, es plausible imaginar, aunque sea hiperbólicamente, que más información se perdería por la derogación o por la limitación sustancial en el Acta de Acceso a la Información, en todas las leyes de transparencia locales, estatales y federales, y en todas las leyes y decisiones de la Corte Suprema sobre juicios públicos ${ }^{78}$-la gran mayoría de ellas criaturas de los 60 y 70- que por la derogación de la misma Primera Enmienda. En términos menos hiperbólicos, el punto es que las libertades negativas protegidas por la Primera Enmienda -la libertad frente a restricciones estatales- pueden ser menos importantes, o al menos no más importantes para promover los valores que subyacen a la Primera Enmienda que aquellas políticas públicas y condiciones sociales que promueven el conocimiento y que, a pesar de ello, son sustantivamente diversas de aquellas libertades negativas. Si pensamos, por ejemplo, que, siguiendo a Mill, es esencial desafiar ideas aceptadas como una forma de avanzar en el conocimiento o evitar la autocomplacencia intelectual, entonces es importante no solo proteger a los desafiantes sino también asegurarse de que existen estos desafiantes, incluso al punto de crearlos -como lo hace la Iglesia católica con el abogado del diablo- y, por tanto, es necesario adoptar pasos positivos para crear aquellas instituciones que garanticen que existan en los hechos aquellos desafíos ${ }^{79}$. De igual forma, si es importante para la democracia que exista un robusto debate público en materias de políticas públicas, entonces las políticas que realmente promueven o incluso crean tal debate no son menos importantes que la preocupación por no restringir a aquellos que, por su propia iniciativa, terminan siendo los participantes del debate.

Con el mismo efecto, en consecuencia, si hay valor en la información sobre el gobierno y si hay un valor en controlar los abusos del Estado ${ }^{80}$, entonces las diversas políticas -no los derechos constitucionales- encarnadas en leyes de acceso a la información, leyes de reuniones abiertas, requisitos de divulgación o publicidad activa y muchos otros pueden ser un componente tan valioso para servir a aquellas finalidades como la misma protección de expositores o editores que pueden estar criticando al gobierno. En otras

${ }^{78}$ V. gr., Richmond Newspapers, Inc. v. Virginia, 448 U.S. 555, 580 (1980).

${ }^{79} \mathrm{Y}$ Mill reconoce el punto, pese a que sus seguidores y quienes lo citan, raramente lo hacen. Véase Mill, supra note 59, at 42-43 (instando a que incluso donde hay posiciones asentadas y no existan visiones desafiantes, varios "artificios" por vía de contestación deben ser empleados con el objeto de asegurar que "la inteligente y viviente aprehensión de la verdad” no sea impedida).

${ }^{80}$ Vincent Blasi, The Checking Value in First Amendment Theory, 1977 Am. B. Found. Res. J. 521. mucho de K.r. Popper, 1 The open Society and its Enemies (5th ed. 1966) igualmente gira en torno a la relativa importancia de prevenir malos gobiernos de realizar conductas negativas, incluso al costo de hacer más difícil a los buenos gobiernos hacer cosas positivas. Popper también señala que la "tradicional pregunta de la teoría política, ‘Quién debe gobernar?’ debe ser reemplazada por una pregunta completamente distinta del tenor de “Cómo podemos organizar nuestras instituciones políticas de manera que gobernantes malos o incompetentes... no puedan causar tanto daño?'”. Karl r. Popper, conjectures and refutations: the growth of Scientific Knowledge 25 (3d ed. 1969). 
palabras, la libertad de expresión puede ser mejor entendida como un componente, y quizás ni siquiera el más importante, de un compromiso más amplio con la transparencia.

No es mi intención con esto retraerme sobre mis anteriores observaciones acerca de pensar la transparencia en términos de teoría de las decisiones. La transparencia no debe ser entendida como un valor que necesariamente debe ser maximizado a expensas de otros intereses. Ciertamente sigue siendo importante reconocer los daños a las buenas decisiones que la transparencia, como mecanismo de protección frente a malas decisiones, puede en ocasiones causar. En cualquier caso, lo que pretendo sugerir es que los aspectos de la transparencia que parte de quienes estudiamos libertad de expresión o Derecho Constitucional hemos relegado a las bajas regiones del Derecho Administrativo, Derecho Local o, simplemente, al reino de las políticas públicas -libre de las limitaciones constitucionales o legales- pueden ser, de hecho, más importantes para los propósitos perseguidos por la Primera Enmienda que la doctrina de la Primera Enmienda en sí misma.

En el análisis final, sin embargo, no es mi objetivo aquí adoptar una posición nítida a favor o en contra de más o menos transparencia gubernamental que la que actualmente tenemos en los Estados Unidos. Parte de la razón de esta ambivalencia es la innegable naturaleza contingente y contextual del valor de la transparencia. Y parte de la razón es que mi propio estilo intelectual va más en la dirección de la clarificación conceptual, que pienso que tenemos poco, que en la dirección de una acentuada prescripción normativa, de lo cual pienso tenemos demasiado. Por tanto, gran parte de mi justificación para evitar conclusiones considerables sobre el cuándo y el dónde necesitamos más o menos transparencia en Estados Unidos -tengo otras ideas sobre Corea del Norte, Zimbawe e incluso China- es también mi creencia que no podemos participar de manera razonable en estos debates a menos que primero clarifiquemos de qué es lo que estamos hablando, cuáles son los desafíos y cómo debemos conducirnos en estas discusiones. Si esta Clase ha contribuido en tal dirección, entonces he sido exitoso y agradezco a la familia Baum, a la Facultad de Derecho y al público presente la oportunidad de hacer precisamente eso.

\section{BIBLIOGRAFÍA}

Ackermann, John R., “Toward Open Source Hardware”, en University of Dayton Law Review, Vol. 34, 2009.

Alexander, Larry A., "Constitutionalism”, en Golding, Martin \& Edmundson, William (eds.), The Blackwell Guide to the Philosophy of Law and Legal Theory, Blackwell, Malden, 2005.

ARTICle 19, "Changing the Climate for Freedom of Expression and Freedom of Information", 2009 [En Línea] http://www.article19.org/pdfs/publications/changing-the-climate-forfreedom-of-expression-and-freedom-of-information.pdf

ArTiCle 19, "The London Declaration for Transparency, the Free Flow of Information and Development" 2010 [En Línea] http:// www.right2info-mdgs.org/wp-content/uploads/ London-Declaration.pdf

BArber, Sotirios A., "Welfare and the Instrumental Constitution", en American Jouornal of Jurisprudence Vol. 42, 1997. 
Bartlett, John, Familiar Quotations, Little, Brown and Co., Boston, 12a ed., 1950, p. 111.

Ben-Shahar, Omri \& Schneider, Carl E., "The Failure of Mandated Disclosure", en University of Pennsylvania Law Review Vol. 159, 2011.

BerLIN, Isaiah, "Two Concepts of Liberty", en, el mismo, Four Essays on Liberty, Oxford University Press, Oxford, 1969.

BLASI, Vincent, "Holmes and the Marketplace of Ideas", en Supreme Court Review 2004.

BoK, Sissela, Secrets: On the Ethics of Concealment and Revelation, Oxford University Press, Oxford, 1982.

Boudin, Chesa "Note, Publius and the Petition: Doe v. Reed and the History of Anonymous Speech", en Yale Law Journal Vol. 120, 2011.

Brandeis, Louis, Other People's Money and How the Bankers Use It, Frederick Stokes Co., New York, 1914.

Brown, Robert L., "Just a Matter of Time? Video Cameras at the United States Supreme Court and the State Supreme Courts", en The Journal of Appellate Practice and Process Vol. 9, 2007.

Christensen, Clayton \& Hwang, Jason, "What Obama’s Health Care Team Can Learn from Massachusetts", en Harvard Business Review Blog [En Línea]: http://blogs.hbr.org/hbr/oninnovation/2009/01/what-obamas-health-care-team-c.html

Citron, Danielle Keats, “Open Code Governance”, en University of Chicago Legal Forum Vol. 2008, 2008

Coase, Ronald H., "Lecture, Blackmail”, en Virginia Law Review Vol. 74, 1988.

Coleman, Jr., William T., "Mr. Justice Felix Frankfurter: Civil Libertarian As Lawyer and As Justice: Extent to Which Judicial Responsibilities Affected His Pre-Court Convictions", en Ronald D. Rotunda ed., Six Justices on Civil Rights, Oceana Pub., Nueva York, 1983.

Coglianese, Cary et al., "Transparency and Public Participation in the Federal Rule- making Process: Recommendations for the New Administration”, en George Washington Law Review Vol. 77, 2009.

THE Compact Edition of the Oxford English Dictionary, Oxford University Press, Oxford, Tomo II, 1971.

Countryman, Vern, “Justice Douglas and Freedom of Expression”, en University of Illinois Law Forum Vol. 1978, 1978.

Crocker, Lawrence, Positive Liberty: An Essay in Normative Political Philosophy, Kluwer, Dordretch, 1980.

Crovitz, L. Gordon, "Op.-Ed., Transparency is More Powerful Than Regulation”, en The Wall Street Journal Mar. 30, 2009, A21.

Dolinar, Richard \& Leininger, S. Luke, "Pay for Performance or Compliance? A Second Opinion on Medicare Reimbursement", en Indiana Health Law Review Vol. 3, 2006, pp. 418-20.

Epstein, Richard A., "Blackmail, Inc.", en University of Chicago Law Review Vol. 50, 1983.

Estlund, Cynthia, "Just the Facts: The Case for Workplace Transparency", en Stanford Law review, Vol. 63, 2011.

Etzioni, Amitai, "Is Transparency the Best Disinfectant?", en Journal of Political Philosophy Vol. 18, 2010.

Feinberg, Kenneth, "Transparency and Civil Justice: The Internal and External Value of Sunlight", en DePaul Law Review, Vol. 58, 2009.

Fenster, Mark, "Designing Transparency: The 9/11 Commission and Institutional Form”, en Washington \& Lee Law Review Vol. 65, 2008.

Fenster, Mark, "Seeing the State: Transparency As Metaphor", en Administrative Law Review Vol. 62, 2010.

Ferrell, Allen, "The Case for Mandatory Disclosure in Securities Regulation Around the World", en Brooklyn Journal of Corporate, Financial \& Commercial Law Vol. 2, 2007. 
Fidell, Eugene R., “Transparency, 2009”, en Hastings Law Journal Vol. 61, 2009.

Frank, John P., "Hugo L. Black: Free Speech and the Declaration of Independence", en University of Illinois Law Forum Vol. 1977, 1977.

Fortney, Katie, "Ending Copyright Claims in State Primary Legal Materials: Toward an Open Source Legal System", en Law Library Journal Vol. 102, 2010.

Fung, Archon et al., Full Disclosure: The Perils and Promise of Transparency, Cambridge University Press, Cambirdge, 2007.

Funk, William, "Public Participation and Transparency in Administrative Law-Three Examples As an Object Lesson", en Administrative Law Review Vol. 61, 2009.

Garrett, Elizabeth et al., "Constitutional Issues Raised by the Lobbying Disclosure Act", en Luneburg, William V. et al. (eds.) The Lobbying Manual: A Complete Guide to Federal Lobbying Law and Practice, American Bar Association, 4a ed., 2009.

Gersen, Jacob E. \& O'Connell, Anne Joseph, "Hiding in Plain Sight? Timing and Transparency in the Administrative State", en University of Chicago Law Review Vol. 76, 2009.

Gilmore, Grant, "Some Reflections on Oliver Wendell Holmes, Jr.", en Green Bag 2d Vol. 2, 1999.

Goldman, Alvin I. \& Cox, James C., "Speech, Truth, and the Free Market for Ideas", en Legal Theory Vol. 2, 1996, pp. 2-3.

Goldring, Gary F., "Note, Mandatory Disclosure of Corporate Projections and the Goals of Securities Regulation”, en Columbia Law Review Vol. 81, 1981.

Golobay, Diana, "Fed Publishes Wave of Rules for Mortgage Origination Transparency", en Housingwire [En Línea] http://www.housingwire.com/2010/08/16/ fed_publishes_wave_of_rules_for_mortgage_origination_transparency.

Grexa, Thomas, "Title VII Tenure Litigation in the Academy and Academic Freedom-A Current Appraisal”, en Dickinson Law Review Vol. 96, 1991.

Griffin, Oren R., "Constructing a Legal and Managerial Paradigm Applicable to the ModernDay Safety and Security Challenge at Colleges and Universities", en Saint Louis University Law Journal Vol. 54, 2009.

Havighurst, Clark C., "Regulations of Health Facilities and Services by 'Certificate of Need'”, en Virginia Law Review Vol. 59, 1973.

Hermalin, Benjamin E. \& Weisbach, Michael S., "Transparency and Corporate Governance (Univ. of Cal. \& Nat'l Bureau of Econ. Research, Working Paper No. W12875, 2007)" [En Línea] http://papers.ssrn.com/sol3/papers.cfm?abstract_id $=958628$

Hood, C. \& Heald, D. (eds.), Transparency: The Key to Better Governance?, Oxford University Press, Oxford, 2006.

Ingber, Stanley, "The Marketplace of Ideas: A Legitimizing Myth", en Duke Law Journal Vol. 1984, 1984.

JIN, Ginger Zhe \& Leslie, Phillip, "The Effect of Information on Product Quality: Evidence from Restaurant Hygiene Grade Cards”, en Quarterly Journal of Economics Vol 118, 2003.

Johnson, Nicholas, "Open Meetings and Closed Minds: Another Road to the Mountaintop", en Drake Law Review Vol. 53, 2004.

Kreimer, Seth F., "The Freedom of Information Act and the Ecology of Transparency", en University of Pennsylvania Journal of Constitutional Law Vol. 10, 2008.

Kurland, Philip B., "Justice Robert H. Jackson-Impact on Civil Rights and Civil Liberties", en University of Illinois Law Forum Vol. 1977.

Lathrop, Daniel \& Ruma, Laurel (eds.), Open Government: Collaboration, Transparency, and Participation, O'Reilly, Cambridge, 2010.

Leghorn, Joseph et al., "The First Amendment and FDA Restrictions on Off-Label Uses: The Call for a New Approach", en Food \& Drug Law Journal Vol. 63, 2008. 
Levy, Ken, "The Solution to the Real Blackmail Paradox: The Common Link Between Blackmail and Other Criminal Threats", en Connecticut Law Review Vol. 39, 2007.

LoPucki, Lynn M., "Court-System Transparency”, en Iowa Law Review Vol. 94, 2009.

Lukacs, John, Democracy and Populism: Fear and Hatred, Yale University Press, New Haven, 2005.

Madison, Michael J. et al., "Constructing Commons in the Cultural Environment", en Cornell Law Review Vol. 95, 2010.

Marks, Jonathan H., "9/11 + 3/11 + 7/7 = ?: What Counts in Counterterrorism", en Columbia Human Rights Law Review Vol. 37, 2006.

Marotta-Wurgler, Florencia “Does Disclosure Matter? (NYU Law and Economics, Research Paper No. 10-54, 2010)" [En Línea] http://papers.ssrn.com/sol3/papers.cfm?abstract_id=1713860

Marshall, William P., "In Defense of the Search for Truth As a First Amendment Justification", en Georgia Law Review Vol. 30, 1995.

Michelman, Frank I., "Foreword: On Protecting the Poor Through the Fourteenth Amendment", en Harvard Law Review Vol. 83, 1969.

Mikva, Abner J., "Justice Brennan and the Political Process: Assessing the Legacy of Baker v. Carr", en University of Illinois Law Review Vol. 1995 (3), 1995.

MiLl, John Stuart, On Liberty, W.W. Norton \& Co. Inc., 1975 (1859).

Milton, John, A reopagitica: A Speech for the Liberty of Unlicensed Printing, Macmillan, 1959 (1644).

Nathanson, Nathaniel L., "The Philosophy of Mr. Justice Brandeis and Civil Liberties Today", en University of Illinois Law Forum Vol. 1979, 1979.

National Freedom of Information Coalition, "State FOI Laws" [En Línea] http://www.nfoic. org/state-foi-laws

National Security Archive, "Sunshine and Shadows: The Clear Obama Message for Freedom of Information Meets Mixed Results" [En Línea] http://www.gwu.edu/ nsarchiv/NSAEBB/ NSAEBB308/index.htm

Nelson, William, On Justifying Democracy, Routledge and Kegan Paul, Londres, 1980.

Note, "Open Meeting Statutes: The Press Fights for the 'Right to Know', en Harvard Law Review Vol. 75, 1962.

O'Connor, John F. \& Baratz, Michael, "Some Assembly Required: The Application of State Open Meeting Laws to Email Correspondence", en George Mason Law Review Vol. 12, 2004.

Pozen, David E., "Deep Secrecy", en Stanford Law Review, Vol. 62, 2010.

Ripken, Susanna Kim, "The Dangers and Drawbacks of the Disclosure Antidote: Toward a More Sub- stantive Approach to Securities Regulation", en Baylor Law review Vol. 58, 2006.

Rock, Edward, "Securities Regulation As Lobster Trap: A Credible Commitment Theory of Mandatory Disclosure", en Cardozo Law Review Vol. 23, 2002.

Rothberg, Michael B. et al., "Choosing the Best Hospital: The Limitations of Public Quality Reporting”, en Health Affairs Vol. 27, 2008.

SACHS, Albie, "Enforcement of Social and Economic Rights", en American University International Law Review Vol. 22, 2007.

Samaha, Adam M., "Government Secrets, Constitutional Law, and Platforms for Judicial Intervention", en UCLA Law Review Vol. 53, 2006.

SAVEr, Richard S., "At the End of the Clinical Trial: Does Access to Investigational Technology End As Well?", en Western New England Law Review Vol. 31, 2009.

Schatffer, Stephen, "Comment, Sunshine in Cyberspace: Electronic Deliberation and the Reach of Open Meeting Laws”, en Saint Louis University Law Journal Vol. 48, 2004.

Schauer, Frederick, Free Speech: A Philosphical Enquiry, Cambridge University Press, Cambridge, 1982, pp. 15-17.

SCHauer, Frederick, "Parsing the Pentagon Papers", paper no publicado, Joan Shorenstein Center, Harvard University, John F. Kennedy School of Government, 1991. 
Schauer, Frederick "Is It Better to Be Safe Than Sorry?: Free Speech and the Precautionary Principle", en Pepperdine Law Review Vol. 36, 2009.

SCHAUER, Frederick, "Facts and the First Amendment", en UCLA Law Review Vol. 57, 2010.

Siebecker, Michael R., "Trust \& Transparency: Promoting Efficient Corporate Disclosure Through Fiduciary-Based Discourse”, en Washington University Law Review Vol. 87, 2009.

Simon, Carol J., "The Effect of the 1933 Securities Act on Investor Information and the Performance of New Issues", en American Economic Review Vol. 79, 1989.

Sмiтh, Henry E., "The Harm in Blackmail”, en Northwestern University Law Review Vol. 92, 1998.

Solum, Lawrence B., "Legal Theory Lexicon: Transparency", en Legal Theory Blog, [En Línea] http://lsolum.typepad.com/legaltheory/2010/10/legal-theory-lexicon-transparency. html.

STANGer, Allison, One Nation under Contract: The Outsourcing of American Power and the Future of Foreign Policy, Yale University Press, New Haven, 2009.

Stewart, Daxton R. "Chip", "Let the Sunshine in, or Else: An Examination of the "Teeth" of State and Federal Open Meetings and Open Records Laws", en Communication Law and Policy Vol. 15, 2010.

Symposium, "Blackmail and Other Forms of Arm-Twisting", en University of Pennsylvania Law Review Vol. 141, 1993.

Symposium, “Tradeoffs of Candor: Does Judicial Transparency Erode Legitimacy?”, en New York University Annual Survey of American Law Vol. 64, 2009.

Thaler, Richard H. \& Sunstein, Cass R., "Op.-Ed., Disclosure is the Best Kind of Credit Regulation”, en The Wall Street Journal, Ago. 13, 2008, A17.

Transparency International, “Corruption Perceptions Index 2010 Results”, [En Línea] http:// www.transparency.org/policy_research/surveys_indices/cpi/2010/results

Tushnet, Mark V., "The Jurisprudence of Thurgood Marshall”, en University of Illinois Law Review Vol. 1996 (4), 1996.

U.S. Department of Justice, "Freedom of Information Act reference Guide (2010)" [En Línea] http://www.justice.gov/oip/referenceguide.htm

Vetter, Greg R., "Open Source Licensing and Scattering Opportunism in Software Standards", en Boston College Law Review Vol. 48, 2007.

Whelan, Timothy P., "New York's Open Meetings Law: Revision of the Political Caucus Exemption and Its Implications for Local Government", en Brooklyn Law Review Vol. 60, 1995.

Wiles, Ellen, "Aspirational Principles or Enforceable Rights?: The Future for Socio-Economic Rights in National Law", en American University International Law Review Vol. 22, 2006. 